\title{
CONSECUTIVE EVALUATION OF EULER SUMS
}

\section{KHRISTO N. BOYADZHIEV}

Received 12 June 2001

We describe a simple method for a consecutive evaluation of the Euler sums $S(r, p), r=$ $1,2, \ldots$ in terms of zeta values.

2000 Mathematics Subject Classification: 40A25, 40B05, 11M06, 33E99.

1. Introduction. The Euler sums

$$
S(r, p)=\sum_{n=1}^{\infty} \frac{H_{n}^{(r)}}{n^{p}},
$$

where

$$
H_{n}^{(r)}=\frac{1}{1^{r}}+\frac{1}{2^{r}}+\cdots+\frac{1}{n^{r}}, \quad r \geq 1, p \geq 2
$$

have enjoyed considerable attention in a number of publications during the last decade (see $[1,3,5,6,7,8])$. For the history of the problem see, for instance, the comments in $[1,2,5,7]$. Euler [6, pages 217-264] evaluated $S(1, p)$ and several $S(r, p)$ in terms of zeta values, where

$$
\xi(p)=\sum_{n=1}^{\infty} \frac{1}{n^{p}}, \quad p>1,
$$

is the Riemann zeta function. The numbers $S(r, p)$ for $p+r$ odd were explicitly evaluated for the first time in [3]. As commented in [3], there is strong evidence that for $p+r$ even $S(r, p)$ can be evaluated only in some particular cases: $S(p, p), S(2,4), S(4,2)$. A powerful method for evaluation of Euler sums, based on the residue theorem, was presented in [7].

The purpose of this note is to describe an elementary procedure for consecutive evaluation of $S(r, p), r=1,2,3, \ldots$, by a recursive relation. The method requires only general knowledge of differential calculus. We hope that this technique will give more insight into the nature of these numbers and make them more accessible. For instance, it is explicitly shown that when both $p$ and $r$ are odd, $S(p, r)$ can be evaluated in terms of zeta values and $S(k, l)$ where both $k, l$ are even and $k+l=p+r$.

We first list some notations and simple facts. As Euler found:

$$
S(r, p)+S(p, r)=\zeta(p) \zeta(r)+\zeta(p+r)
$$

and therefore,

$$
S(p, p)=\left(\frac{1}{2}\right)\left[\zeta(p)^{2}+\zeta(2 p)\right], \quad p \geq 2 .
$$


We have

$$
\begin{gathered}
w(p, r) \equiv \sum_{n, m=1}^{\infty} \frac{1}{n^{p}(n+m)^{r}}=\zeta(p) \zeta(r)-S(r, p), \\
w(p) \equiv \sum_{n, m=1}^{\infty} \frac{1}{n^{p-1} m(n+m)}=S(1, p) .
\end{gathered}
$$

Define also

$$
v(p, r) \equiv \sum_{n, m=1}^{\infty} \frac{1}{n^{p}(n-m)^{r}}
$$

(the "prime" in the sigma means $m \neq n$ ). We easily find that

$$
v(p, r)=S(r, p)-\zeta(p+r)+(-1)^{r} \zeta(p) \zeta(r) .
$$

Analogous to (1.7),

$$
\nu(p) \equiv \sum_{n, m=1}^{\infty} \frac{1}{n^{p-1} m(n-m)}=S(1, p)-2 \zeta(p+1) .
$$

All these relations are evaluated by simple direct computations. For example,

$$
\begin{aligned}
v(p) & =\sum^{\prime} \frac{1}{n^{p}} \frac{n}{m(n-m)}=\sum_{n=1}^{\infty} \frac{1}{n^{p}} \sum_{m=1}^{\infty}\left(\frac{1}{m}+\frac{1}{n-m}\right) \\
& =\sum_{n=1}^{\infty} \frac{1}{n^{p}}\left(H_{n}^{(1)}-\frac{2}{n}\right)=S(1, p)-2 \zeta(p+1) .
\end{aligned}
$$

2. Evaluation of $S(r, p), r=1,2,3$. We use an idea from [4]. Set

$$
\sigma_{+}(p ; x) \equiv \sum_{n=1}^{\infty} \frac{1}{n^{p}(n+x)} .
$$

Let $m$ be an arbitrary positive integer. Summing for all $n=1,2, \ldots, n \neq m$, define also

$$
\sigma_{-}(p ; x ; m) \equiv \sum_{n=1}^{\infty} \frac{1}{n^{p}(n-x)} .
$$

LEMMA 2.1. For every $x$ where the equations are defined:

$$
\begin{aligned}
\sigma_{+}(p ; x) & =\sum_{k=1}^{p-1} \frac{(-1)^{k-1} \zeta(p-k+1)}{x^{k}}+\frac{(-1)^{p-1}}{x^{p}} \sum_{n=1}^{\infty} \frac{x}{n(n+x)}, \\
\sigma_{-}(p ; x ; m) & =-\sum_{k=1}^{p-1} \frac{1}{x^{k}}\left[\zeta(p-k+1)-\frac{1}{m^{p-k+1}}\right]+\frac{1}{x^{p}} \sum^{\prime} \frac{x}{n(n-x)} .
\end{aligned}
$$


Proof.

$$
\sigma_{-}=-\frac{1}{x} \sum^{\prime} \frac{n-x-n}{n^{p}(n-x)}=-\frac{1}{x} \sum^{\prime} \frac{1}{n^{p}}+\frac{1}{x} \sum^{\prime} \frac{1}{n^{p-1}(n-x)}
$$

The first sum on the right is

$$
\sum^{\prime} \frac{1}{n^{p}}=\zeta(p)-\frac{1}{m^{p}}
$$

and repeating this procedure $p-2$ more times we obtain (2.4). Equation (2.3) is demonstrated by the same way (see [4]).

We can now immediately evaluate $S(1, p)$. Write (2.4) with $p-1$ in place of $p$

$$
\sum_{n=1}^{\infty} \frac{1}{n^{p-1}(n-x)}=-\sum_{k=1}^{p-2} \frac{1}{x^{k}}\left[\zeta(p-k)-\frac{1}{m^{p-k}}\right]+\frac{1}{x^{p-1}} \sum_{n=1}^{\infty} \frac{x}{n(n-x)} .
$$

Then set $x=m$, divide both sides by $m$, and sum for $m=1,2, \ldots$ to get

$$
v(p)=-\sum_{k=1}^{p-2}[\zeta(k+1) \zeta(p-k)-\zeta(p+1)]+\sum_{n, m=1}^{\prime} \frac{1}{m^{p-1} n(n-m)} .
$$

The second sum on the right is, in fact, $-v(p)$. Solving for $v(p)$ and using (1.10) we find Euler's formula

$$
S(1, p)=\left(1+\frac{p}{2}\right) \zeta(p+1)-\frac{1}{2} \sum_{k=1}^{p-2} \zeta(k+1) \zeta(p-k),
$$

for every $p>2$. When $p=2$ the sum is missing and $S(1,2)=2 \zeta(3)$.

Using Lemma 2.1, we can evaluate $S(r, p), r=2$, by differentiating for $x$ in (2.3) and summing for $x=m$. Thus

$$
\begin{aligned}
\frac{d}{d x} \sigma_{+}(p ; x)= & -\sum_{n=1}^{\infty} \frac{1}{n^{p}(n+x)^{2}} \\
= & -\sum_{k=1}^{p-1} \frac{(-1)^{k-1} k \zeta(p-k+1)}{x^{k+1}} \\
& -\frac{(-1)^{p-1} p}{x^{p+1}} \sum_{n=1}^{\infty} \frac{x}{n(n+x)}+\frac{(-1)^{p-1}}{x^{p}} \sum_{n=1}^{\infty} \frac{1}{(n+x)^{2}} .
\end{aligned}
$$

For differentiation we use that

$$
\frac{x}{n(n+x)}=\frac{1}{n}-\frac{1}{n+x}
$$

Setting $x=m$, summing over $m$ and taking into account (1.6) and (1.7) we come to

$$
\begin{aligned}
S(2, p)-\zeta(2) \zeta(p)= & -\sum_{k=1}^{p-1}(-1)^{k-1} k \zeta(k+1) \zeta(p-k+1) \\
& -(-1)^{p-1} p S(1, p+1)+(-1)^{p-1}[\zeta(2) \zeta(p)-S(2, p)] .
\end{aligned}
$$


Solving for $S(2, p)$ we find

$$
\begin{aligned}
S(2, p)\left[1-(-1)^{p}\right]= & {\left[1-(-1)^{p}\right] \zeta(2) \zeta(p) } \\
& -\sum_{k=1}^{p-1}(-1)^{k-1} k \zeta(k+1) \zeta(p-k+1)-(-1)^{p-1} p S(1, p+1) .
\end{aligned}
$$

When $p$ is odd, we can express $S(2, p)$ in terms of zeta values as

$$
S(2, p)=2 \zeta(2) \zeta(p)-\frac{1}{2} \sum_{k=1}^{p-1}(-1)^{k-1} k \zeta(k+1) \zeta(p-k+1)-\frac{1}{2}(-1)^{p-1} p S(1, p+1),
$$

combined with (2.9) this is (cf. [3])

$$
\begin{aligned}
S(2, p)= & \frac{1}{4} \sum_{k=1}^{p-1}\left(2(-1)^{k} k+p\right) \zeta(k+1) \zeta(p-k+1) \\
& +\zeta(2) \zeta(p)-\frac{1}{4}\left(3 p+p^{2}\right) \zeta(p+2) .
\end{aligned}
$$

For $p$ even, the left-hand side in (2.13) is zero and we come to an equivalent form of (2.9).

We now continue this process and try to evaluate $S(3, p)$ from $d^{2} \sigma_{+} / d x^{2}$

$$
\begin{aligned}
\left(\frac{d}{d x}\right)^{2} \sigma_{+}(p ; x)= & 2 \sum_{n=1}^{\infty} \frac{1}{n^{p}(n+x)^{3}} \\
= & \sum_{k=1}^{p-1} \frac{(-1)^{k-1} k(k+1) \zeta(p-k+1)}{x^{k+2}}+\frac{(-1)^{p-1} p(p+1)}{x^{p+2}} \sum_{n=1}^{\infty} \frac{x}{n(n+x)} \\
& -2 \frac{(-1)^{p-1} p}{x^{p+1}} \sum_{n=1}^{\infty} \frac{1}{(n+x)^{2}}-2 \frac{(-1)^{p-1}}{x^{p}} \sum_{n=1}^{\infty} \frac{1}{(n+x)^{3}},
\end{aligned}
$$

which implies $(x=m$ and summing over $m)$

$$
\begin{aligned}
S(3, p)\left[1-(-1)^{p}\right]= & {\left[1-(-1)^{p}\right] \zeta(3) \zeta(p) } \\
& -\frac{1}{2} \sum_{k=1}^{p-1}(-1)^{k-1} k(k+1) \zeta(k+2) \zeta(p-k+1) \\
& -\frac{1}{2}(-1)^{p-1} p(p+1) S(1, p+2) \\
& +(-1)^{p-1} p[\zeta(2) \zeta(p+1)-S(2, p+1)] .
\end{aligned}
$$

When $p$ is odd, we can express $S(3, p)$ in terms of zeta values and $S(2, p+1)$, where $p+1$ is even

$$
\begin{aligned}
S(3, p)= & \zeta(3) \zeta(p)-\frac{1}{4} \sum_{k=1}^{p-1}(-1)^{k-1} k(k+1) \zeta(k+2) \zeta(p-k+1) \\
& -\frac{p(p+1)}{4} S(1, p+2)+\frac{p}{2}[\zeta(2) \zeta(p+1)-S(2, p+1)] .
\end{aligned}
$$


For instance, when $p=3$

$$
S(3,3)=2 \zeta(3)^{2}-\frac{3}{2} S(2,4)
$$

which in view of (1.5) gives

$$
S(2,4)=\zeta(3)^{2}-\frac{1}{3} \zeta(6)=\zeta(3)^{2}-\frac{\pi^{6}}{2835} .
$$

When $p=5$, we find

$$
S(3,5)=5 \zeta(3) \zeta(5)-\frac{\pi^{2}}{3600}-\frac{5}{2} S(2,6) .
$$

Several such relations are listed in [7, page 23]. The exceptional character of $S(2,4)$ is clear from (2.19): we can evaluate it, because we can evaluate $S(3,3)$. The numbers $S(2, q)$ for even $q>4$ are resisting evaluation in terms of zeta values.

When $p$ is even, the left-hand side in (2.17) is zero and the equation turns into (2.15). In order to evaluate $S(3, p)$ in this case, we use the expression $\sigma_{-}$from (2.4). Consecutive differentiation gives

$$
\begin{aligned}
\left(\frac{d}{d x}\right)^{2} \sigma_{-}= & 2 \sum^{\prime} \frac{1}{n^{p}(n-x)^{3}} \\
= & -\sum_{k=1}^{p-1} \frac{k(k+1)}{x^{k+2}}\left[\zeta(p-k+1)-\frac{1}{m^{p-k+1}}\right] \\
& +\frac{p(p+1)}{x^{p+2}} \sum^{\prime} \frac{x}{n(n-x)}-\frac{2 p}{x^{p+1}} \sum^{\prime} \frac{1}{(n-x)^{2}}+\frac{2}{x^{p}} \sum^{\prime} \frac{1}{(n-x)^{3}} .
\end{aligned}
$$

Note that

$$
\frac{x}{n(n-x)}=\frac{1}{n-x}-\frac{1}{n}
$$

Setting $x=m$, summing over $m$, and taking into consideration (1.9), we come to

$$
\begin{aligned}
S(3, p)= & \zeta(3) \zeta(p)+\left(1+\frac{p^{3}+5 p}{12}\right) \zeta(p+3) \\
& -\frac{1}{4} \sum_{k=1}^{p-1} k(k+1) \zeta(k+2) \zeta(p-k+1) \\
& -\frac{1}{4} p(p+1) S(1, p+2)-\frac{1}{2}[S(2, p+1)+\zeta(p+1) \zeta(2)] \quad(p \text { even }) .
\end{aligned}
$$

Here $p+1$ is odd, so $S(2, p+1)$ is a combination of zeta values-see (2.15), and therefore $S(3, p)$ is a combination of zeta values.

3. General recursive formulas. Applying the above method for ascending values of $r$, the sums $S(r, p)$ can be evaluated in terms of zeta values for all $r+p$ odd: when $r$ is even, we use consecutive derivatives of $\sigma_{+}(p ; x)$-see [4, Lemma 2]. We obtain 
representations of the form (2.13) where we can solve for $S(r, p)$ in case $p$ is odd. When $r$ is odd, we use the derivatives of $\sigma_{-}(p ; x ; m)$ as shown above. The general representations are listed in the following theorem.

THEOREM 3.1. With the interpretation $\zeta(1)=0$, we have

$$
\begin{aligned}
S(r, p)= & \zeta(r) \zeta(p)+\frac{1}{2} \sum_{k=0}^{p-2}(-1)^{k+1}\left(\begin{array}{c}
r+k-1 \\
r-1
\end{array}\right) \zeta(p-k) \zeta(r+k) \\
+\frac{1}{2} \frac{(-1)^{p-1}}{2(p-1) !} \sum_{k=0}^{r-2} & \frac{(r+p-k-2) !}{(r-k-1) !} \\
& \times[S(k+1, r+p-k-1)-\zeta(k+1) \zeta(r+p-k-1)]
\end{aligned}
$$

for $p$ odd and all $r$. Also

$$
\begin{aligned}
S(r, p)= & \zeta(p+r)-\zeta(r) \zeta(p) \\
& -\frac{1}{2} \sum_{k=0}^{p-2}(-1)^{k+1}\left(\begin{array}{c}
r+k-1 \\
r-1
\end{array}\right)[\zeta(p-k) \zeta(r+k)-\zeta(p+r)] \\
& +\frac{1}{2(p-1) !} \sum_{k=0}^{r-2}(-1)^{k} \frac{(r+p-k-2) !}{(r-k-1) !}[S(k+1, r+p-k-1)-\zeta(p+r) \\
& \left.+(-1)^{k+1} \zeta(k+1) \zeta(r+p-k-1)\right]
\end{aligned}
$$

for $r$ odd and every $p$.

Equation (3.1) is derived from (2.3) (details can be found in [4]) and (3.2) follows the same way from (2.4). Equation (3.1) was possibly found for the first time by Nielsen [9, page 48, equation (12)].

Every sum $S(r, p)$ can be expressed in terms of zeta values and $S(n, m)$, where $n+m=p+r$ and $n<r$. When $p+r$ is odd, (3.1) and (3.2) working together bring to the representation of $S(r, p)$ in terms of zeta values. The explicit formula can be found in [3] or [7]. When $r$ and $p$ are both odd, $S(r, p)$ can be expressed only in terms of zeta values and $S(n, m)$, where $n+m=p+r$ and $n, m$ are both even (see also the comments in [3, Section 3] and [7, page 23]).

\section{REFERENCES}

[1] D. H. Bailey, J. M. Borwein, and R. Girgensohn, Experimental evaluation of Euler sums, Experiment. Math. 3 (1994), no. 1, 17-30.

[2] B. C. Berndt, Ramanujan's Notebooks. Part I, Springer-Verlag, New York, 1985.

[3] D. Borwein, J. M. Borwein, and R. Girgensohn, Explicit evaluation of Euler sums, Proc. Edinburgh Math. Soc. (2) 38 (1995), no. 2, 277-294.

[4] K. N. Boyadzhiev, Evaluation of Euler-Zagier sums, Int. J. Math. Math. Sci. 27 (2001), no. 7, 407-412.

[5] R. E. Crandall and J. P. Buhler, On the evaluation of Euler sums, Experiment. Math. 3 (1994), no. $4,275-285$.

[6] L. Euler, Opera Omnia, vol. 15, Teubner, Berlin, 1917.

[7] P. Flajolet and B. Salvy, Euler sums and contour integral representations, Experiment. Math. 7 (1998), no. 1, 15-35. 
[8] J. G. Huard, K. S. Williams, and N.-Y. Zhang, On Tornheim's double series, Acta Arith. 75 (1996), no. 2, 105-117.

[9] N. Nielsen, Die Gammafunktion. Band I. Handbuch der Theorie der Gammafunktion. Band II. Theorie des Integrallogarithmus und verwandter Transzendenten, Chelsea, New York, 1965 (German).

Khristo N. Boyadzhiev: Department of MAthematics, OHio Northern University, ADA, OH 45810, USA

E-mail address: k-boyadzhiev@onu.edu 


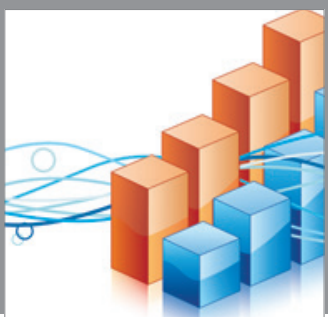

Advances in

Operations Research

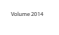

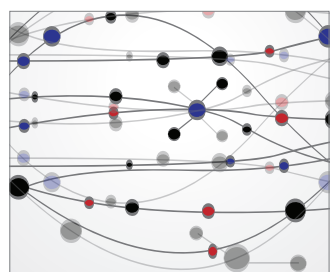

\section{The Scientific} World Journal
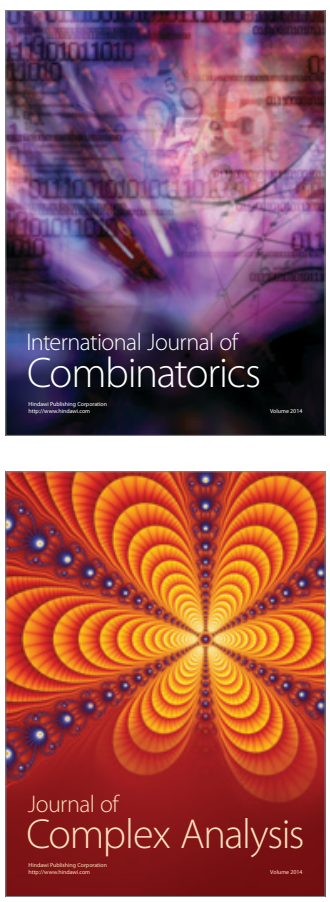

International Journal of

Mathematics and

Mathematical

Sciences
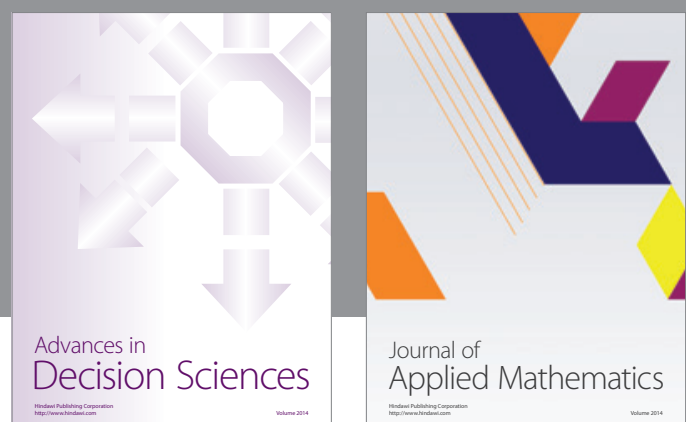

Journal of

Applied Mathematics
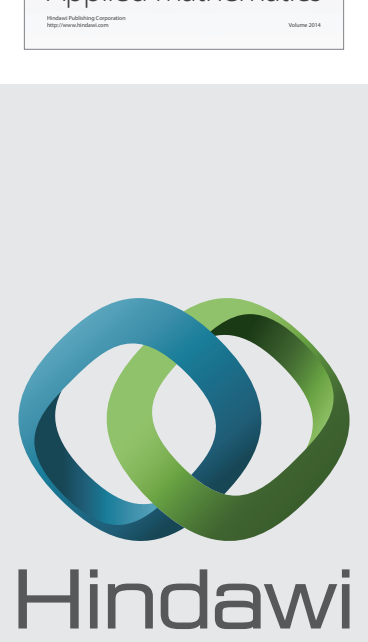

Submit your manuscripts at http://www.hindawi.com
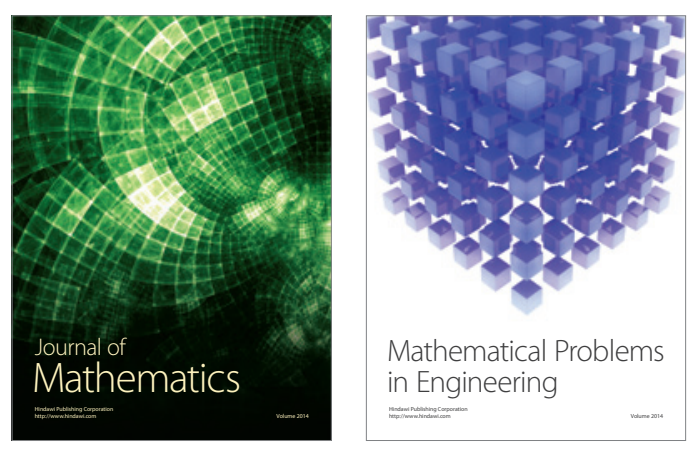

Mathematical Problems in Engineering
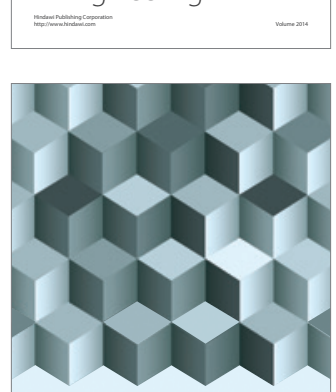

Journal of

Function Spaces
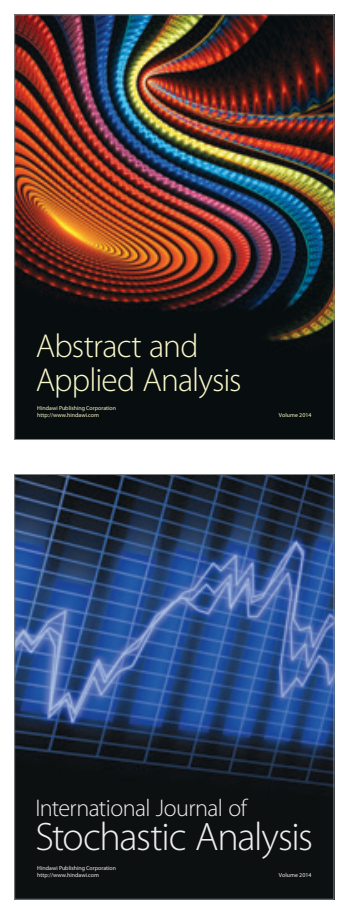

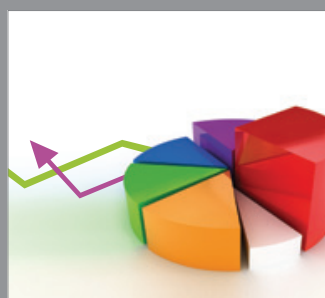

ournal of

Probability and Statistics

Promensencen
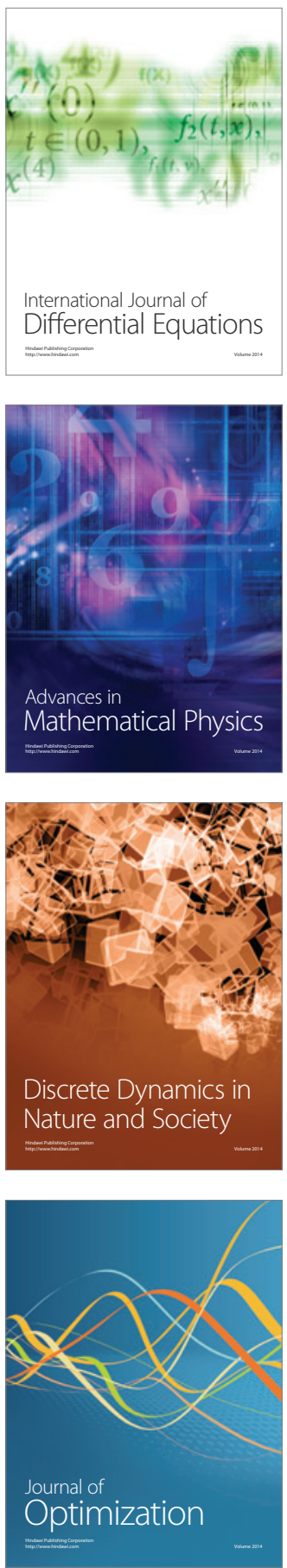